\title{
Brain metastasis affecting frontal lobe misdiagnosed as bipolar disorder, without any neuroimaging exam: two case reports with fatal outcomes
}

\author{
João Gama Marques ${ }^{1,2 *}$ (6)
}

Clínica Universitária de Psiquiatria e Psicologia Médica, Faculdade de Medicina, Universidade de Lisboa, Lisboa, Portugal

Consulta de Esquizofrenia Resistente, Hospital Júlio de Matos, Centro Hospitalar Psiquiátrico de Lisboa, Lisboa, Portugal

Received 16 November 2018; Accepted 18 December 2018

Key words: Cancer, brain metastasis, neuropsychiatry, organic psychosis, bipolar.

\section{To the Editor}

It was with great pleasure that we recently read an article in your journal, regarding psychiatric, behavioral, and cognitive disorders in patients with extracranial cancers. ${ }^{1}$ This fine article reminded us of two patients of ours, both admitted in our ward for psychiatric symptoms secondary to extracranial cancers with undiagnosed brain metastasis.

Case Report 1: A 70-year-old woman was seen in an outpatient psychiatric consultation setting after 1 week of mood elevation, sexual disinhibition, and delusional misidentification syndrome. She was being medicated with sertraline and mirtazapine for depression, plus anastrozole for breast cancer relapse prevention. The patient was admitted in our psychiatric hospital for the treatment of a bipolar manic episode. We found cognitive impairment with amnesia, disorientation, dysgraphia, and dyscalculia. Blood work, urine tests, and chest radiography were normal. Electroencephalography was diffusely slow, suggesting encephalic dysfunction. A brain computerized tomography (Figure 1(a)) disclosed left temporal and occipital intra-axial expansive lesions. Admitting psychiatric disorder secondary to breast cancer with brain metastasis, the patient was transferred to a general hospital, but passed away before any neurosurgical procedure was performed.

Case Report 2: A 40-year-old woman was seen in the emergency room after 1 week of having a depressed mood with delusions of ruin, agitation, and suicidal ideation. In the last 25 years, she had been treated with different types of medication for drug abuse and personality disorder. The patient was admitted in our psychiatric hospital for the treatment of bipolar mixed state. We found ataxia, apathy,

*Address correspondence to: João Gama Marques, Hospital Júlio de Matos, Centro Hospitalar Psiquiátrico de Lisboa, Avenida Brasil, 53, Lisboa 1749-002, Portugal. (Email: joaogamamarques@gmail.com) and disorganized behavior. Her blood work and urine tests were normal. The chest radiography suggested a $5 \mathrm{~cm}$ nodule in the upper lobe of the right lung, highly suggestive of pulmonary neoplasm. Electroencephalography was diffusely slow, suggesting encephalic dysfunction. The computerized tomography brain scan (Figure 1(b)) revealed masses at subcortical left frontal and periventricular right occipital locations. Admitting psychiatric disorder secondary to lung cancer with brain metastasis, the patient was transferred to a general hospital, but passed away before any neurosurgical procedure was performed.

Since the 16th century, with the practice of performing human autopsies for academic purposes, brain tumors have been associated with mental symptoms. Classic studies have shown how psychiatric symptoms have been overlooked for the diagnosis of brain tumors, with many neurologically silent brain tumors being accidentally detected during psychiatric evaluation. More recently, Madhusoodanan et al. have been publishing important papers regarding this problem, ${ }^{2}$ bringing international attention to the topic of psychiatric symptoms secondary to brain tumors.

There are two types of brain tumors: primary (up to $75 \%$ ) and secondary (up to $25 \%$ ), the latter being more often associated with neuropsychiatric symptoms; although there may be an association between location and psychiatric symptoms, it is difficult to predict the symptoms based on the location or vice versa. Some symptoms of brain lesions may depend on the functions of the networks underlying the affected areas, with significant association being found between mood symptoms and frontal tumors, as probably happened with both of our patients. But more importantly, we should always be aware of the following: 


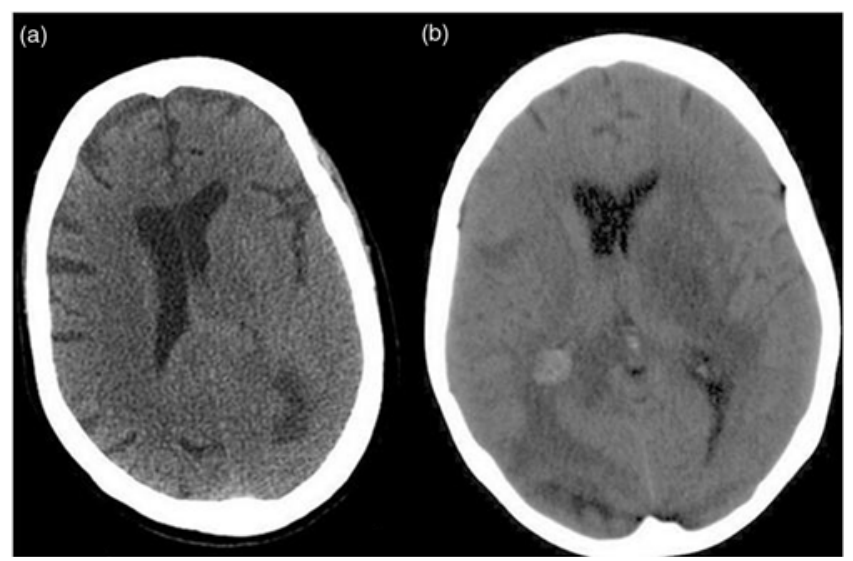

FIGURE 1. Brain metastasis leading to bipolar disorder misdiagnosis: (a) left temporal and occipital intra-axial expansive lesions; (b) subcortical left frontal and periventricular right occipital expansive lesions.

- $\quad$ Psychiatric symptoms secondary to brain tumors may respond to psychotropic medications;

- $\quad$ Primary brain tumors may occur 20 times more frequently in psychiatric patients;

- There is no clinical method of excluding a brain tumor by its psychiatric manifestations;

- Psychiatric symptoms in brain tumors can mimic clinical presentation of psychiatric disorders ${ }^{3}$;

- Circa $13 \%$ of intracranial tumors may contribute to anamnesis only with mental disturbances;

- Only $15 \%$ of the patients with undiagnosed brain tumors did not present psychiatric symptoms. ${ }^{4}$

Despite all the available evidence, we still find undiagnosed brain tumors in psychotic patients coming to us, referred for psychiatric care, so two questions echo in our head:

1. Why are we neglecting the necessary medical work-up of first-episode psychosis?
2. Why in the age of brain scans is a brain tumor still mistaken for a psychiatric illness ${ }^{5}$

\section{Disclosure}

João Gama Marques has nothing to disclose.

\section{REFERENCES:}

1. Pravettoni G, Dell'Osso B, Bocci T, et al. Psychiatric, behavioral, and cognitive disorders in patients with extracranial cancers. CNS Spectr. 2018; 23: 1-14.

2. Madhusoodanan $\mathrm{S}$, Ting $\mathrm{MB}$, Farah $\mathrm{T}$, et al. Psychiatric aspects of brain tumors: a review. World J Psychiatry. 2015; 5(3): 273-285.

3. Ristić DI, Vesna P, Sanja P, et al. Brain tumors in patients primarily treated psychiatrically. Vojnosanit Pregl. 2011; 68(9): 809-814.

4. Mardaga S, Al Bassir M, Bracke J, et al. Which psychiatric symptoms must raise suspicion about a possible brain tumor? Rev Med Liege. 2017; 72(9): 399-405

5. Caruso R, Piro A. Why in the age of CT scans and MRIs is a brain tumour mistaken for a psychiatric illness? BMJ Case Rep. 2017; 4: 2017. 